\title{
Low-temperature and ammonia-free epitaxy of GaN/AIGaN/GaN heterostructure
}

D.M. Tobaldi, ${ }^{1, *}$ V. Triminì, ${ }^{1}$ A. Cretì, ${ }^{2}$ M. Lomascolo, ${ }^{2}$ S. Dicorato, ${ }^{3}$ M. Losurdo,,${ }^{3, *}$ A. Passaseo,,${ }^{1}$ and V. Tasco ${ }^{1}$

${ }^{1}$ CNR-Nanotec, Nanotechnology Institute, Via Monteroni 73100, Lecce, Italy

${ }^{2}$ CNR-IMM, Institute for Microelectronic and Microsystems, Via Monteroni 73100, Lecce, Italy

${ }^{3}$ CNR-Nanotec, Nanotechnology Institute, c/o Dipartimento di Chimica - Università di Bari, via Orabona 4, 70126, Bari, Italy

*Corresponding authors.

E-mail addresses: david.tobaldi@nanotec.cnr.it; david@davidtobaldi.org (DM Tobaldi);

maria.losurdo@cnr.it (M Losurdo)

Twitter: @D14MT (DM Tobaldi) 


\begin{abstract}
Wide band gap semiconductors are very attractive because of their broad applications as electronics and optoelectronics materials - GaN-based materials being by far the most promising. For the production of such nitride-based optical and power devices, metal-organic chemical vapour deposition (MOCVD) is routinely used. However, this has disadvantages, such as the large consumption of ammonia gas, and the need for high growth temperature. To go beyond such a limit, in this study we successfully developed a remote plasma assisted MOCVD (RPA-MOCVD) approach for the epitaxial growth of high-quality GaN/AIGaN heterostructures on $4 \mathrm{H}-\mathrm{SiC}$ substrates. Our RPA-MOCVD has the advantages of lower growth temperature $\left(750{ }^{\circ} \mathrm{C}\right.$ ) compared to conventional MOCVD route, and the use of a remote $\mathrm{N}_{2} / \mathrm{H}_{2}$ plasma instead of ammonia for nitrides growth, generating in situ the $\mathrm{NH}_{x}(x=0-3)$ species needed for the growth. As assessed by structural, morphological, optical and electrical characterisation, the proposed strategy provides an overall cost-effective and green approach for high-quality GaN/AIGaN heteroepitaxy, suitable for high electron mobility transistors (HEMT) technology.
\end{abstract}

\title{
1. Introduction
}

III-nitrides have been intensively studied in view of their interesting applications as electronics and optoelectronics components, including light-emitting diodes (LEDs), laser-diodes (LDs), ${ }^{1,2}$ and highpower/high frequency high electron mobility transistors (HEMTs). ${ }^{3}$ GaN-based devices, primarily fabricated on foreign substrates because of the lack of high quality and large area GaN substrates, exploit the semiconductor technology reference deposition techniques of molecular beam epitaxy (MBE), and metal organic chemical vapour deposition (MOCVD). These techniques are characterised by significant production costs, as they require, respectively, either ultra-high vacuum conditions, or energy consuming procedures involving high growth temperatures (in the $1000{ }^{\circ} \mathrm{C}$ range), ${ }^{4}$ and large flows of critical handling gases like ammonia $\left(\mathrm{NH}_{3}\right)$. It is thus essential to re-think the aspects of the processes used to make thin films for such technologies to a green and sustainable "philosophy", as also recently pointed out by Pedersen et al. ${ }^{5}$ Indeed, in the last years, novel approaches have been studied for the synthesis of such materials, allowing for example, very fast growth rates, ${ }^{6,7}$ or lower deposition temperatures. ${ }^{8,9}$ Among them, plasma technologies represent a high-potential solution for $\mathrm{NH}_{3}$ replacement with nitrogen, $\mathrm{N}_{2}$, and nitrogen/hydrogen, $\mathrm{N}_{2} / \mathrm{H}_{2}$ mixtures, and for growth temperature reduction.

In this work, we propose a remote plasma-assisted MOCVD (RPA-MOCVD) approach, which revealed itself in high quality, low-temperature growth of GaN/AIGaN heterostructure on silicon carbide. The peculiarity of our RP is that only neutrals and electronically excited $\mathrm{NH}_{x}(x=0-3)$ radicals, and $\mathrm{N}$-atoms vibrationally excited interact with the growth surface, avoiding ion and electron bombardment, as the substrate is positioned in the plasma afterglow. The presence of the RP source, fed by $\mathrm{H}_{2}$ and $\mathrm{N}_{2}$, also grants the advantage of operating 
the low temperature $\left(200^{\circ} \mathrm{C}\right)$ steps of SiC cleaning and nitridation in situ. This happens before starting the growth at a temperature of $750{ }^{\circ} \mathrm{C}$, with plasma generated $\mathrm{NH}_{x}$ species in situ - instead of ammonia as nitrogen source. Such a process is an efficient and low-cost means to make high-quality GaN/AIGaN heterostructure in a more sustainable way as compared to conventional semiconductor epitaxial growth techniques, having potential applications in GaN-based electronics. ${ }^{10-16}$

\section{Methods}

\subsection{Growth of GaN/AIGaN epilayers}

The structure of the sample is reported in Figure 1a: the AIN buffer layer, grown at two different temperatures onto the SiC-4H substrate, is followed by a thick GaN layer $(1.5 \mu \mathrm{m})$, the AlGaN barrier ( $25 \mathrm{~nm}$ ) and a thin GaN cap $(10 \mathrm{~nm}) \cdot{ }^{17}$ Sample preparation and growth were performed in the reactor schematically shown in Figure $1 \mathrm{~b}$. The $4 \mathrm{H}-\mathrm{SiC}$ substrate was preliminarily cleaned in situ by an optimised remote $\mathrm{H}_{2}$ plasma treatment. ${ }^{12}$ Subsequently, a SiC nitridation step was run at $200{ }^{\circ} \mathrm{C}$ switching to a remote $\mathrm{N}_{2}$ plasma to prepare the surface to the AIN nucleation layer. ${ }^{14} \mathrm{~A}$ two-step AIN buffer layer was adopted by growing first a nucleation AIN layer at $200^{\circ} \mathrm{C}$ (thickness of about $80 \mathrm{~nm}$ ), and then increasing the temperature up to $750{ }^{\circ} \mathrm{C}$, using a $\mathrm{N}_{2} / \mathrm{H}_{2}$ plasma and trimethylaluminium (TMA) as Al precursor. The following GaN/AIGaN/GaN layers of the HEMT heterostructure (Figure 1b) were grown at the same temperature using trimethylgallium (TMG) and (TMA) as gallium and aluminium precursor respectively, in $\mathrm{H}_{2}$ carrier gas, and in a remote radiofrequency (RF) $\mathrm{N}_{2} / \mathrm{H}_{2}\left(10 \% \mathrm{H}_{2}\right)$ plasma at the RF power of $200 \mathrm{~W}$, and at a pressure of 5 Torr. The remote plasma configuration was used to have active dissociated atomic nitrogen species avoiding radiative and ion bombardment damage of the growing surface. $\mathrm{H}_{2}$ was also added to react with the $\mathrm{N}$-radicals and form in situ the $\mathrm{NH}_{x}$ radicals needed to remove carbon from the growing surface.
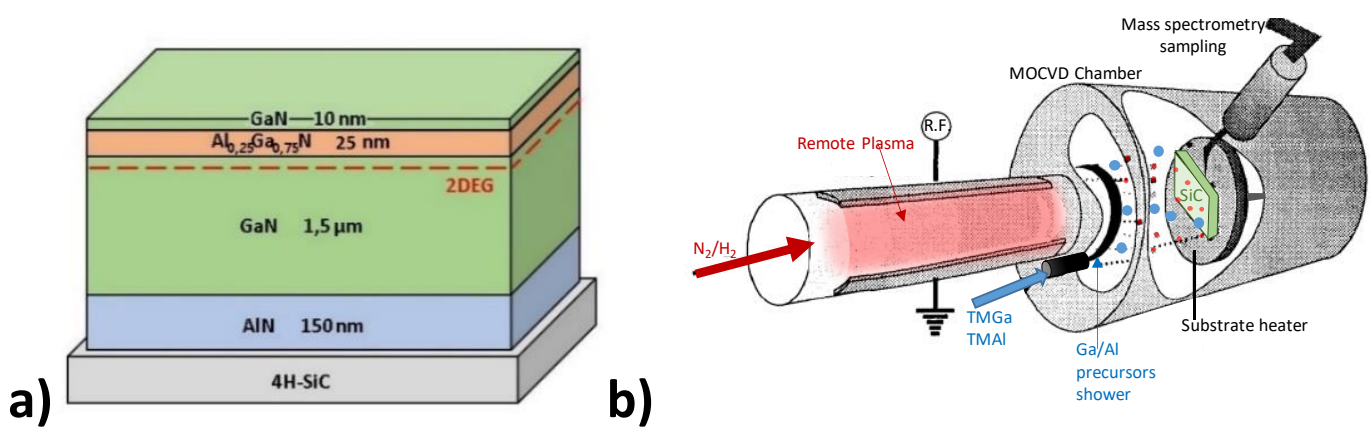

Figure 1 - a) Layout of the GaN/AIGaN/GaN heterostructure grown by RPA-MOCVD on 4H-SiC substrate. b) Schematics of the remote plasma deposition system used to grow the sample. 


\subsection{Characterisation}

The structural properties of the GaN/AIGaN epilayers were examined using high-resolution X-ray diffraction (HR-XRD), performed on a Malvern PanAlytical PW 3050/65 X'pert Pro MRD diffractometer (UK) with CuK $\alpha_{\alpha}$ radiation. To attain structural features of prepared samples, $\omega-2 \theta$ patterns and rocking curves (RC) were recorded in double-axis configuration, in parallel beam mode, using a parabolic mirror, and a four bounce $\mathrm{Ge}$ (220) monochromator; the detector was kept at an open detector configuration. Quantitative information about the density of edge $\left(\rho_{\text {edge }}\right)$, and screw $\left(\rho_{\text {screw }}\right)$ dislocations in the GaN epilayer was attained by collecting RC along GaN asymmetric and symmetric reflections. To this aim, we employed the rigorous method proposed by Kaganer and colleagues. ${ }^{18}$ This assumes the line shape of X-ray diffraction profiles of GaN epitaxial layers to be Gaussian only in the central, most intense part of the reflection; the tails obey a power law decay, typically proportional to $\omega^{-3}$. To minimise effects due to wafer curvature, the beam height was restricted for symmetric RC, whereas for skew symmetric $\omega$-scans the beam width was restricted, as suggested by Moram and Vickers. ${ }^{19} \mathrm{X}$-ray reflectivity (XRR) analysis was accomplished to have information about the thickness of the GaN cap layer. XRR data were modelled with the X'pert Reflectivity software suite. The morphology and the 3-D surface roughness of samples were investigated by atomic force microscopy (AFM). Characterisation of the film topography by AFM was assessed in non-contact mode using the Nanosurf EasyScan atomic force microscope at room temperature (RT), using scan rates of $0.4-2 \mu \mathrm{m} . \mathrm{s}^{-1}$ to obtain $256 \times 256$ pixel images.

Photoluminescence $(\mathrm{PL})$ measurements were performed as a function of temperature, placing the sample on the cold finger of a closed cycle helium cryostat, and the temperature was varied from $10 \mathrm{~K}$ to $300 \mathrm{~K}$. PL was excited with He-Ag laser operating @224.3 nm (5.5 eV), and it was recorded through a $0.32 \mathrm{~m}$ Triax monochromator and cooled Si-CCD camera. The laser excitation power was varied among three orders of magnitude, from 0.5 to $50 \mathrm{~mW}$.

Capacitance-Voltage measurements were performed on the as grown sample by using a mercury-probe system (Materials Development Corp, MDC) connected with an Agilent E4980A Precision LCR Meter. Hall measurements were performed by four-point contacts with a Hall EGK HEM-2000 system, at RT and at liquid nitrogen temperature.

\section{Results and discussions}

\subsection{Structural and morphological analyses}

In the $2 \theta-\omega$ scan of the heterostructure, Figure $2 a$, the $(00.2)$ and $(00.4)$ reflections of $\mathrm{GaN}$ are visible at around 34.5 , and $72.8^{\circ} 2 \theta$, respectively, together with the (00.4), and (00.8) reflections belonging to the $4 \mathrm{H}-\mathrm{SiC}$ substrate (at around 35.5 and $75.3^{\circ} 2 \theta$, respectively). The basal plane of AIN is also recognisable 
(around $35.9^{\circ} 2 \theta$ ). The in-plane epitaxial relationship between $\mathrm{GaN}$ epilayer and the $4 \mathrm{H}-\mathrm{SiC}$ substrate is further confirmed by the $\varphi$ scan around the (10.2) GaN reflection, as reported in Figure $2 \mathrm{~b}$. Results of Kaganer's analysis for GaN symmetric and asymmetric reflections are shown in Figure $2 c, d$, in which the fittings between the observed and simulated patterns are reported. The insets of Figure $2 c, d$ display a loglog scale of simulated and recorded patterns. This is to highlight the tail region in the RCs, which reflects the strain fields in the near vicinity of the dislocation lines. As it is seen in Figure $2 c, d$ the slope of both (00.2) symmetric and (10.1) asymmetric reflections follows a $\omega^{-3}$ asymptotic decay, therefore confirming the scattering from pure screw and edge dislocations, respectively. Average densities of edge and screw dislocations are $2.90 \pm 0.10 \times 10^{10} \mathrm{~cm}^{-2}$ and $0.22 \pm 0.04 \times 10^{10} \mathrm{~cm}^{-2}$, respectively. The difference between edge and screw dislocation density is significant, with $\rho_{\text {edge }}$ being much higher than $\rho_{\text {screw }}-$ this condition resembles a MBE growth method, which uses a $\mathrm{N}_{2}$ RF plasma source. ${ }^{20}$

It is interesting to note that the frequently used FWHM method as a measure of the dislocation densities, ${ }^{19}$ underestimates them, as compared to the more rigorous technique proposed by Kaganer. For instance, as also shown in Table 1, with the former, we achieve $2.79 \times 10^{7} \mathrm{~cm}^{-2}$ and $2.72 \times 10^{9} \mathrm{~cm}^{-2}$ for $\rho_{\text {screw }}$ and $\rho_{\text {edge, }}$ respectively. Still, this is a lower value compared to recent literature: Wang et al. on GaN heterostructure, grown via pulsed laser deposition at $750{ }^{\circ} \mathrm{C}$, found $\rho_{\text {screw }}$ to be $6.17 \times 10^{9} \mathrm{~cm}^{-2}$, and $\rho_{\text {edge }}=2.13 \times 10^{10} \mathrm{~cm}^{-2}$ using the FWHM of (00.2), and (10.2) GaN reflections, respectively. ${ }^{9}$ The present low values of dislocation density achieved in our approach are a combination of the use of the remote plasma in all the steps of the growth, starting from the $\mathrm{H}_{2}$ plasma cleaning and $\mathrm{N}_{2}$ plasma nitridation of the substrate, to the use of plasma activated $\mathrm{NH}_{x}(x=0-3)$ for the growth of a two-step AIN buffer layer and GaN/AIGaN at reduced temperature. From the RC fittings we also obtained the adimensional parameter $M$ that describes the dislocation

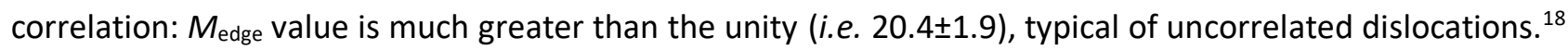
This is also reflected by the high value of characteristic lengths of the dislocation correlations $L$, which are around $1000 \mathrm{~nm}$.

Table 1 - Dislocation density in GaN epitaxial layer grown with conventional growth technologies (MOCVD and MBE), as well as with different plasma enhanced growing methods, assessed with the FWHM method, and with that proposed by Kaganer et al. ${ }^{18}$

\begin{tabular}{llllllll}
\hline $\begin{array}{l}\text { Growth } \\
\text { method }\end{array}$ & $\begin{array}{l}\text { Thickness } \\
(\mu \mathrm{m})\end{array}$ & $\begin{array}{l}\text { Temperature } \\
\left({ }^{\circ} \mathrm{C}\right)\end{array}$ & FWHM & Ref. 15 & FWHM & $\rho_{\text {edgef. }}\left(^{18}\right.$ & Reference \\
\hline MOVPE & 4 & - & - & $2.6 \times 10^{9}$ & - & $1.5 \times 10^{9}$ & 20 \\
HVPE & 5 & - & - & $4.6 \times 10^{10}$ & - & $1.6 \times 10^{9}$ & 20 \\
PAMBE & 2.5 & 690 & - & $5.4 \times 10^{10}$ & - & $5.2 \times 10^{8}$ & 20 \\
PEALD & 0.09 & 425 & - & - & $3.9 \times 10^{7}$ & - & 21 \\
$\begin{array}{l}\text { Pulsed laser } \\
\text { deposition }\end{array}$ & 0.3 & 750 & $2.13 \times 10^{10}$ & - & $6.17 \times 10^{9}$ & - & 9 \\
RPA-MOCVD & 1.5 & 750 & $2.72 \times 10^{9}$ & $2.90 \times 10^{10}$ & $2.79 \times 10^{7}$ & $2.19 \times 10^{9}$ & This work \\
\hline
\end{tabular}




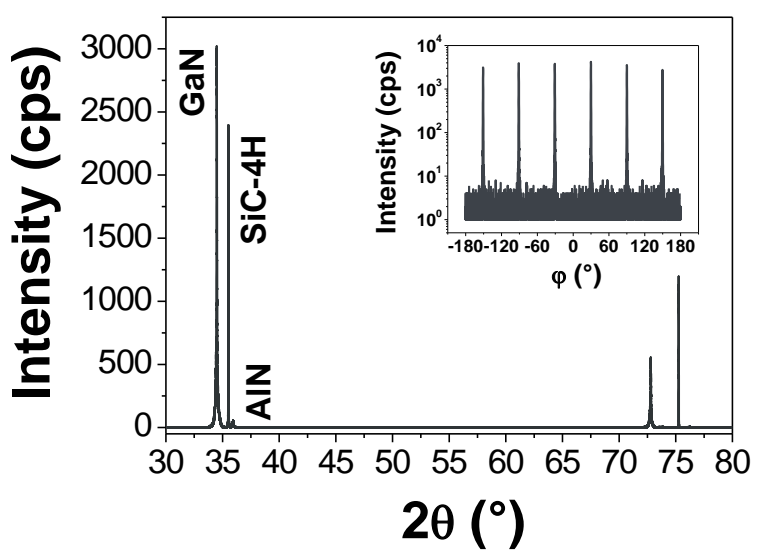

a)

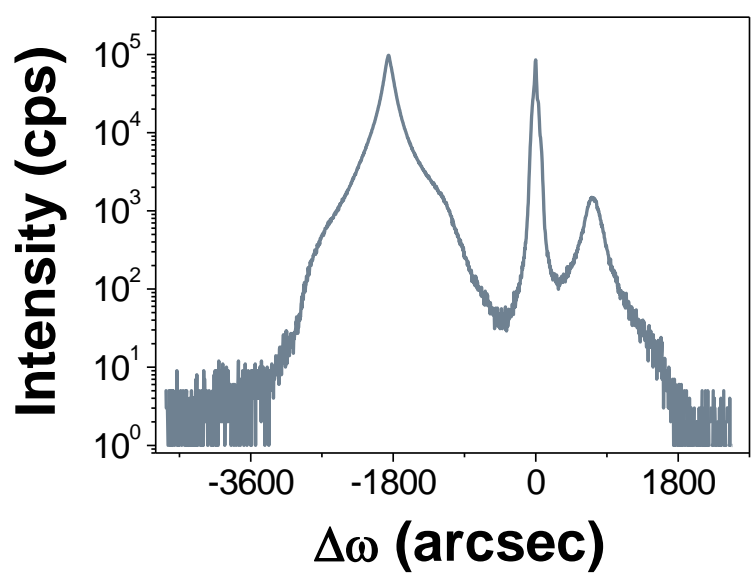

b)

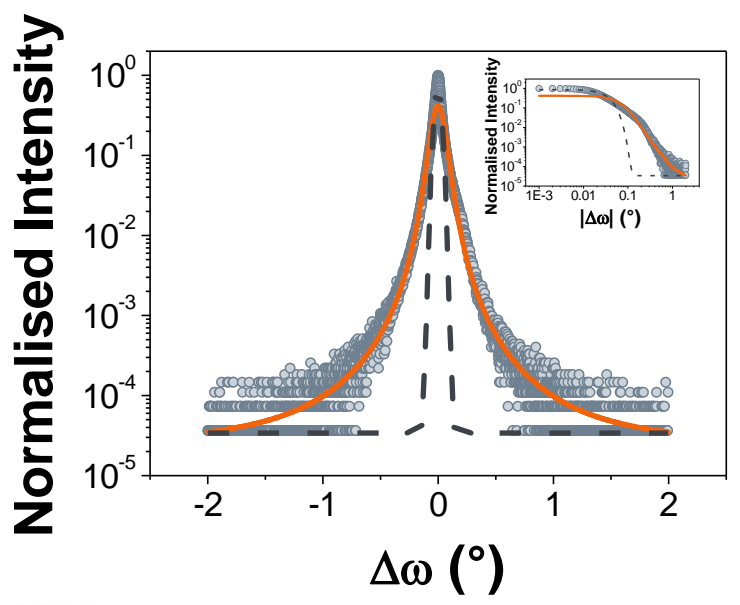

c)

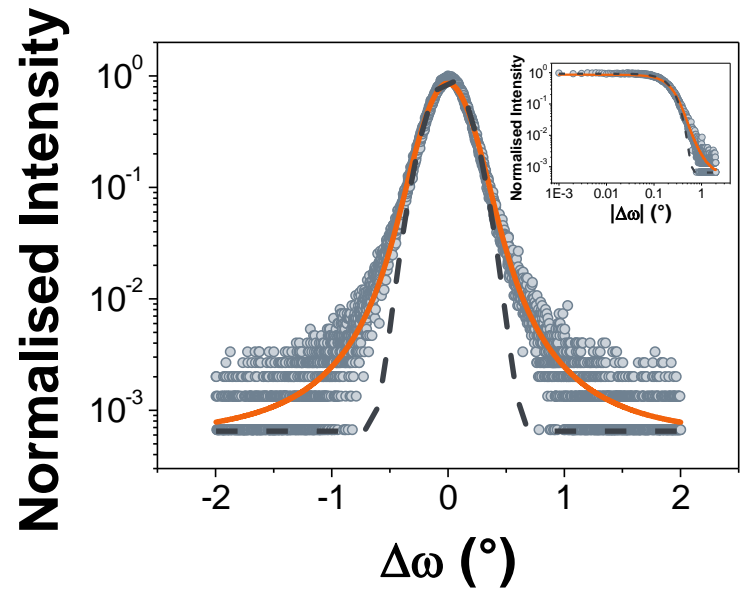

d)

e)

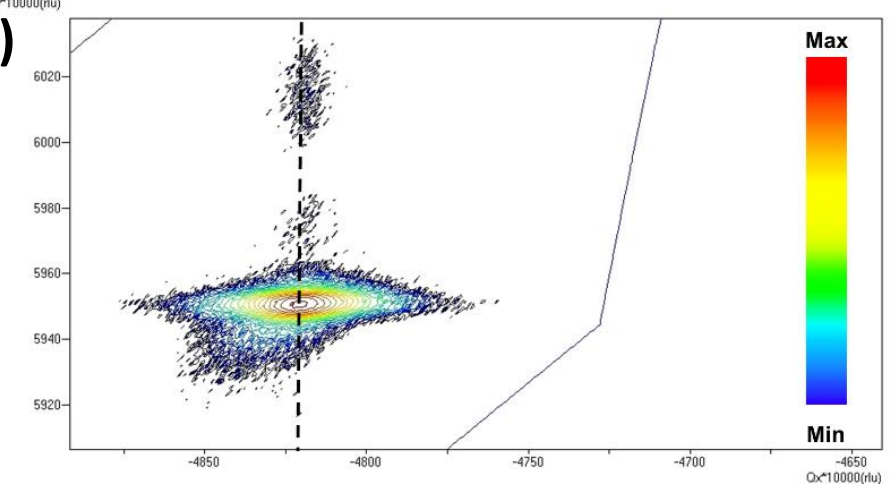

f)

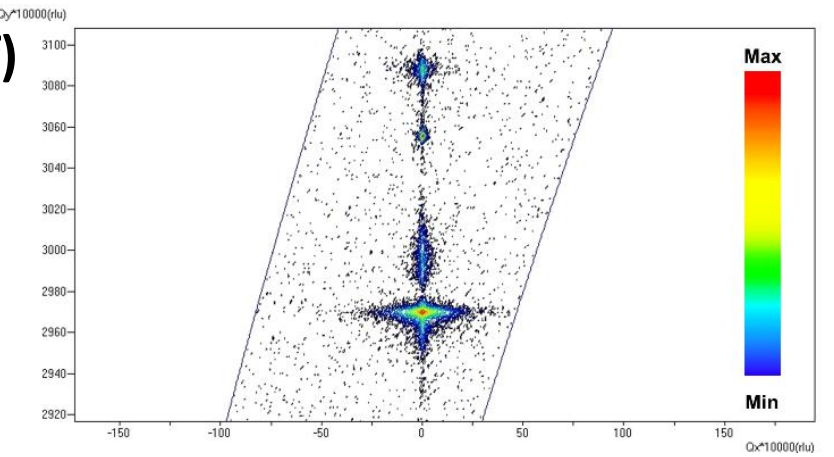

Figure 2 - a) $2 \theta-\omega$ scan of the GaN/AlGaN/GaN heterostructure The inset reports a $\varphi$ scan around the (10.2) asymmetric GaN reflection. b) HRXRD pattern of the sample. c) Rocking curve, collected with an open detector, from GaN (00.2) symmetric reflection. A log-log scale profile to show the $\omega^{-3}$ asymptotic decay is reported in the inset. d) Rocking curve, collected with an open detector, from GaN (10.1) asymmetric reflection. A log-log scale profile to show the $\omega^{-3}$ asymptotic decay is reported in the inset. In c) and d) the light blue circles are the observed data, the continuous orange line the fittings, while the black dotted lines represent a Gaussian profile. e) RSM along the (1) 1.4$)$ reflection. The vertical dashed line represent the position at which a strained AlGaN epilayer should be. f) RSM along the (00.2) reflection.

Reciprocal space maps (RSMs) around the asymmetric ( $\overline{1} \overline{1} .4)$ and symmetric (00.2) reflections were also collected to gain insight on the whole heterostructure (Figure 2e,f). In the (1̄1.4) RSM, the reciprocal lattice points belonging to AIGaN layer are vertically aligned to those from the underlying GaN (Figure 2e). This means that the AIGaN epilayer is partially relaxed over the GaN buffer layer (the black dashed line in Figure 2e shows where the strained AlGaN epilayer should be). The strained in-plane and the out-of-plane unit cell 
parameters from AlGaN, together with those belonging to GaN, were also extracted from the RSMs. From these, values of in- and out-of-plane strain $\left(\varepsilon_{\|}\right.$and $\left.\varepsilon_{\perp}\right)$ of AlGaN were determined according to following equations:22

$\varepsilon_{\|}=\frac{a_{A l G a N}-a_{0}(x)}{a_{0}(x)}$

$\varepsilon_{\perp}=-2 \frac{C_{13}(x)}{C_{33}(x)} \varepsilon_{\|}$

where $a_{\mathrm{AlGaN}}$ and $a_{0}(x)$ are the pseudomorphically strained and bulk in-plane unit cell parameter of AlGaN, respectively. $C_{13}(x)$ and $C_{33}(x)$ are AlGaN elastic constants. Their values were determined by means of a linear interpolation between those of bulk GaN and AIN, which were taken from. ${ }^{23}$ The Al concentration $x$ was determined to be 0.24 by means of Vegard's law, thus being consistent with the nominal value of 0.25 . From these data, we have measured the degree of AIGaN relaxation $r(x)$ according to the method proposed in [24] this being $37 \%$. Eventually, knowing $\varepsilon_{\|}$, the AIGaN in-plane stress $\sigma_{\|}$can be evaluated by using the biaxial modulus $Y^{25}-\varepsilon_{\|}$and $\varepsilon_{\perp}$, and $\sigma_{\|}$of the AlGaN strained epilayer being $0.31 \%,-0.16 \%$, and $1.44 \mathrm{GPa}$, respectively. The lateral coherence length of AIGaN epilayer was found, by X'pert Epitaxy software suite, to be $211 \mathrm{~nm}$, whilst its mosaic spread was 43 arcsec.

XRR pattern of the GaN/AIGaN/GaN heterostructure is displayed in Figure 3. From the fitting, the thickness of the GaN cap-layer has been found to be $11.4 \pm 0.1$. The technique also allows to assess the quality of GaN cap/AIGaN and AIGaN/GaN interfaces, providing roughness values (RMS) of $0.4 \mathrm{~nm}$ and $0.3 \mathrm{~nm}$, respectively. GaN-cap layer RMS, as extracted from the fitting, was of $0.7 \pm 0.1 \mathrm{~nm}$. The representative AFM image of the $\mathrm{GaN} / \mathrm{AlGaN} / \mathrm{GaN}$ heterostructure grown with RPA-MOCVD is shown in Figure $3 \mathrm{~b}$ where the presence of pits due to dislocations, with a density of around $3.6 \times 10^{10} \mathrm{~cm}^{-2}$ can be seen, in agreement with the average dislocation densities resulting from XRD analysis. The root mean square (RMS) roughness over a $1 \mu \mathrm{m} \times 1 \mu \mathrm{m}$ scanning area is $0.36 \mathrm{~nm}$, thus indicating an overall smooth surface. Atomic steps are also observed, suggesting the onset of a step flow growth mode. 
a)

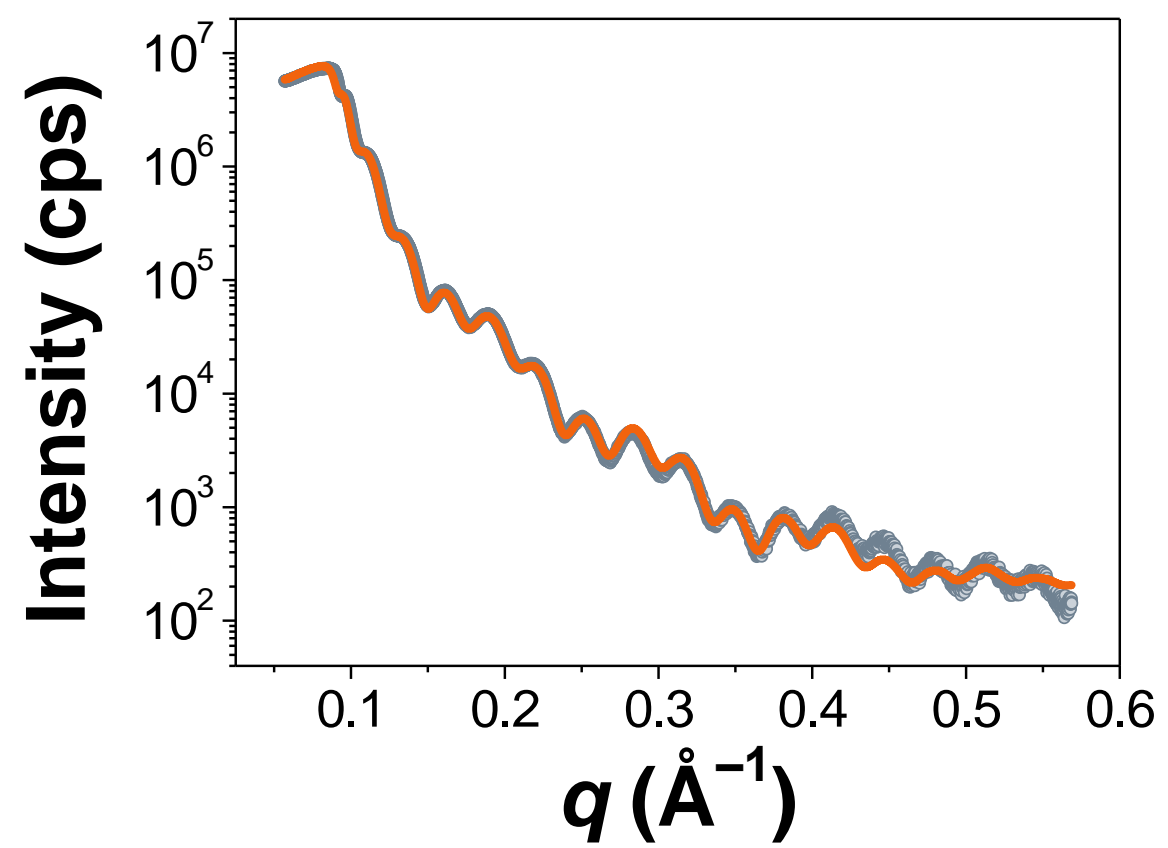

b)

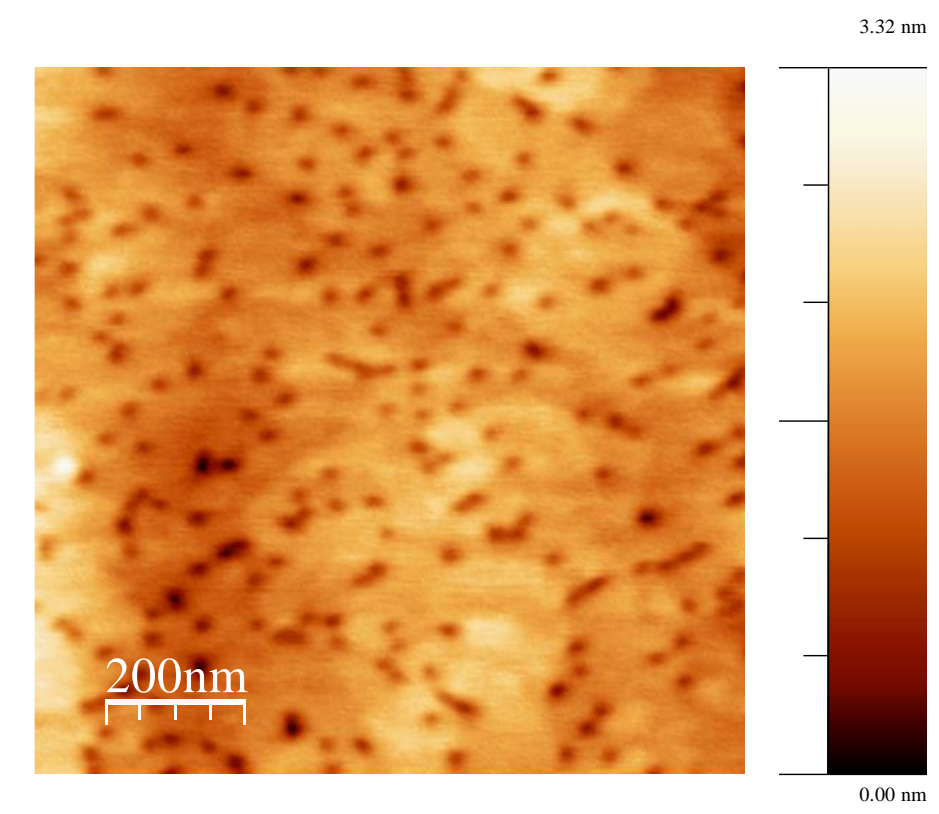

Figure 3 - a) XRR pattern of the GaN/AIGaN/GaN specimen. Full light-blue circles are the observed data, contnuous orange line the calculated data. b) AFM scan over a $1 \mu \mathrm{m} \times 1 \mu \mathrm{m}$ area.

\subsection{Optical characterisation}

PL spectrum recorded at RT (Figure 4a) is dominated by the emission signal originating from the GaN layer at about $3.42 \mathrm{eV}$. At high energy, the contribution of the AIGaN layer is also visible with a narrow emission at $3.88 \mathrm{eV}$, even if with a very low intensity. This allows us to estimate the Al concentration value as $23 \%$ or 
$25 \%$, according to the model of Nepal et al. ${ }^{26}$ or Li et al., ${ }^{27}$ respectively, thus matching the nominal (25\%) and experimental value ( $24 \%$ by $\mathrm{X}$-ray analysis) discussed above.

The GaN band-edge PL signal, attributed to excitonic complexes ( $D^{0} X, A^{0} X$, etc.) and potentially to $2 D E G$ features, typically expected at low temperature and in the sub-band gap of GaN layer, ${ }^{28,29}$ has been investigated by varying the laser power intensity, and the sample temperature.

In Figure $4 b$ the PL spectrum, at the maximum excitation power, is reported, along with the result of a multiGaussian fit performed on the curve, which identifies the contribution of at least 4 sub-bands $\left(x_{1}, x_{2}, x_{3}, x_{4}\right.$ in the Figure). As a general trend, as the excitation power increases we observe, for all $x_{i}$ bands, that the energy peak value does not change (Figure 4c) and that the integrated PL intensity increases almost linearly (on loglog scale, Figure $4 d$ ). Additionally, the $x_{2}$ and $x_{3}$ emission bands show the same slope, suggesting a similar nature of the involved states and/or the engagement of a common state in the transitions. Finally, the $x_{1}$ and $x_{4}$ emission bands disappear at lower excitation intensity $(0.5 \mathrm{~mW})$, and no signature of saturation is present, at least at the maximum power used in the experiments.

Figure $4 \mathrm{e}$ shows the temperature dependence of the energy peak values of $x_{1}, x_{2}, x_{3}$ and $x_{4}$. Up to about 100 $\mathrm{K}$, the peak energy of all bands blue-shifts with the temperature, probably due to delocalisation effects. At high temperatures, $x_{1}$ and $x_{4}$ are no longer visible while $x_{2}$ and $x_{3}$ show the characteristic energy red-shift with the temperature. ${ }^{30}$

The typical experimental energy values reported in literature for GaN excitonic complexes in "strained" AIGaN/GaN heterostructures at low temperature, ${ }^{28,29,31}$ are the following: $\sim 3.47 \mathrm{eV}\left(\mathrm{A}^{0} \mathrm{X}\right.$ neutral acceptor bound), $\sim 3.48 \mathrm{eV}\left(\mathrm{D}^{0} \mathrm{X}\right.$ neutral donor bound), $\sim 3.49 \mathrm{eV}$ ( $\mathrm{FE}_{\mathrm{n}=1}$ free-exciton ground state), $\sim 3.51 \mathrm{eV}\left(\mathrm{FE}_{\mathrm{n}=2}\right.$ freeexciton excited state). Additionally, the 2DEG features (ground and excited states) are reported in a quite spread energy range from $3.44 \mathrm{eV}$ and $3.47 \mathrm{eV} .28,29,31$

Despite their spectral position, the behaviour of $x_{1}$ and $x_{2}$ bands, as a function of excitation power, excludes the involvement of the 2DEG states in the recombination process, since there is no blue-shift evidence with increasing excitation power (that could be from few to hundreds of meV). ${ }^{29}$ Additionally, the $x_{2}$ band can be traced up to $\sim 180 \mathrm{~K}$, well beyond the typical temperature range reported in the literature $(40 \mathrm{~K}-100 \mathrm{~K}) .^{28,29}$ The energy of $x_{3}$ emission band displays the typical thermal energy red-shift for temperature higher than 100 $\mathrm{K}$, and it follows the theoretical curve of free-exciton recombination - at the assumed binding energy value of $23 \mathrm{meV}$ (Figure 4e, dash-dotted line). ${ }^{30}$ Also $x_{2}$ seems to follow this trend, although within a narrower temperature range. Therefore, $x_{2}$ and $x_{3}$ emission bands can be related to the GaN excitonic complex. In particular, $x_{3}$ could be ascribed to the FE (ground state), and $x_{2}$ to the neutral donor bound exciton ( $D^{0} x$ ), which dissociates at high temperature.

Concerning the $x_{1}$ band attribution, since there is no clear evidence of 2DEG - band emission behaviour, as previously discussed, and given its spectral value, we can assume that it originates from transition involving 
neutral acceptor bound exciton $\left(A^{0} X\right)$. Finally, the small contribution observed at the highest spectral energy $\left(x_{4}\right)$, and from $10 \mathrm{~K}$ to $\sim 100 \mathrm{~K}$, could be due to the free exciton in the first excited state.

It is interesting to note that, above the GaN emission, a broad band BB is also observed (Figure $4 \mathrm{f}, \mathrm{g}$ ) and that, at the lowest temperatures (from $10 \mathrm{~K}$ to $90 \mathrm{~K}$ ), a superimposed well-defined narrow band also appears (G). As the temperature increases, the intensity of $G$ band decreases, and, conversely, the BB becomes more and more intense and broadened. For temperature higher than $100 \mathrm{~K}$ the $\mathrm{BB}$ dominates, and the $\mathrm{G}$ band is no more distinguishable (Figure $4 \mathrm{f}, \mathrm{g}$ ). The large width of the BB suggests high trap density in the barrier. Noteworthy, in our experimental conditions (excitation energy is larger than the AIGaN barrier bandgap), PL transitions can also originate from recombination of 2DEG electrons, and free holes of AIGaN, rather than the free ones at GaN valence band, resulting at energies higher than the GaN bandgap. Free holes from AlGaN barrier, moving to the AlGaN/GaN interface can be trapped (localised holes), and involved in 2DEG transitions. ${ }^{29,32,33}$ Therefore, the $\mathrm{G}$ band, observed at high energy, which gradually reduces and disappears at $90 \mathrm{~K}$ (as expected from transitions involving electron in the potential well), can be attributed to the 2DEG contribution.
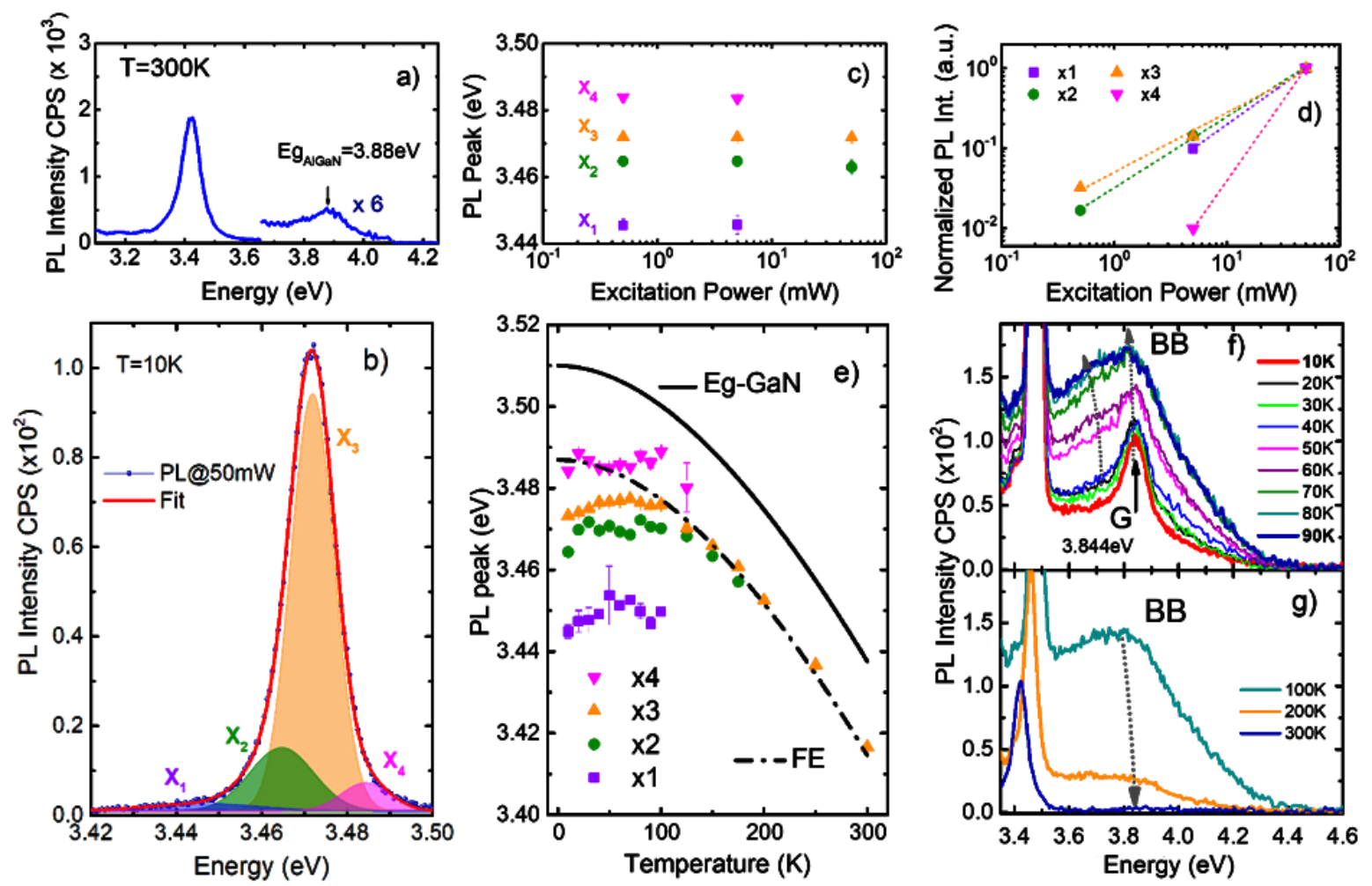

Figure 4 - PL analysis of the GaN/AIGaN/GaN sample: a) RT emission of the structure, the peak from AlGaN is magnified $\times 6$; b) low temperature spectrum of GaN emission, with four bands arising from Gaussian fitting; $c$ ) PL peaks of $x_{1}, x_{2}, x_{3}, x_{4}$ bands as a function excitation power. d) Normalized PL intensity of $x_{1}, x_{2}, x_{3}, x_{4}$ bands as a function excitation power. e) PL peaks of $x_{1}, x_{2}, x_{3}, x_{4}$ bands as a function of temperature. Theoretical curve for GaN bandgap (continuous black line) and free-exciton ground state (dash-dotted line), for binding energy of $23 \mathrm{meV}$, are also shown; f) PL spectra at high energy, as a function of temperature (10 K-90 K). g) PL spectra at high energy, as a function of temperature (100 K-300 K). 


\subsection{Electrical properties}

Capacitance-voltage $(\mathrm{C}-\mathrm{V})$ measurements are performed with the mercury probe to assess the charge profiling in the heterostructure. Fig. 5 shows the $\mathrm{C}-\mathrm{V}$ profile measured at $20 \mathrm{kHz}$, where one can notice the presence of a capacitance plateau due to the 2D electron gas (2DEG) located at the AIGaN/GaN interface and a pinch off voltage of $-6.2 \mathrm{~V}$. A sheet carrier density $N s$ of the $2 \mathrm{DEG}$ of $1.18 \times 10^{13} \mathrm{~cm}^{-2}$ is deduced from the measurement, consistent with the strain and relaxation values estimated by XRD analysis. ${ }^{24}$ The values of the Hall mobility at RT, and at low temperature $\left(77 \mathrm{~K}\right.$ ) are approximately $650 \mathrm{~cm}^{2} \cdot \mathrm{V}^{-1} \mathrm{~cm}^{-1}$ at RT and 1100 $\mathrm{cm}^{2} \cdot \mathrm{V}^{-1} \mathrm{~cm}^{-1}$ at $77 \mathrm{~K},{ }^{34}$ as listed in the inset of Figure 5 , in line with the measured dislocation density. ${ }^{9}$

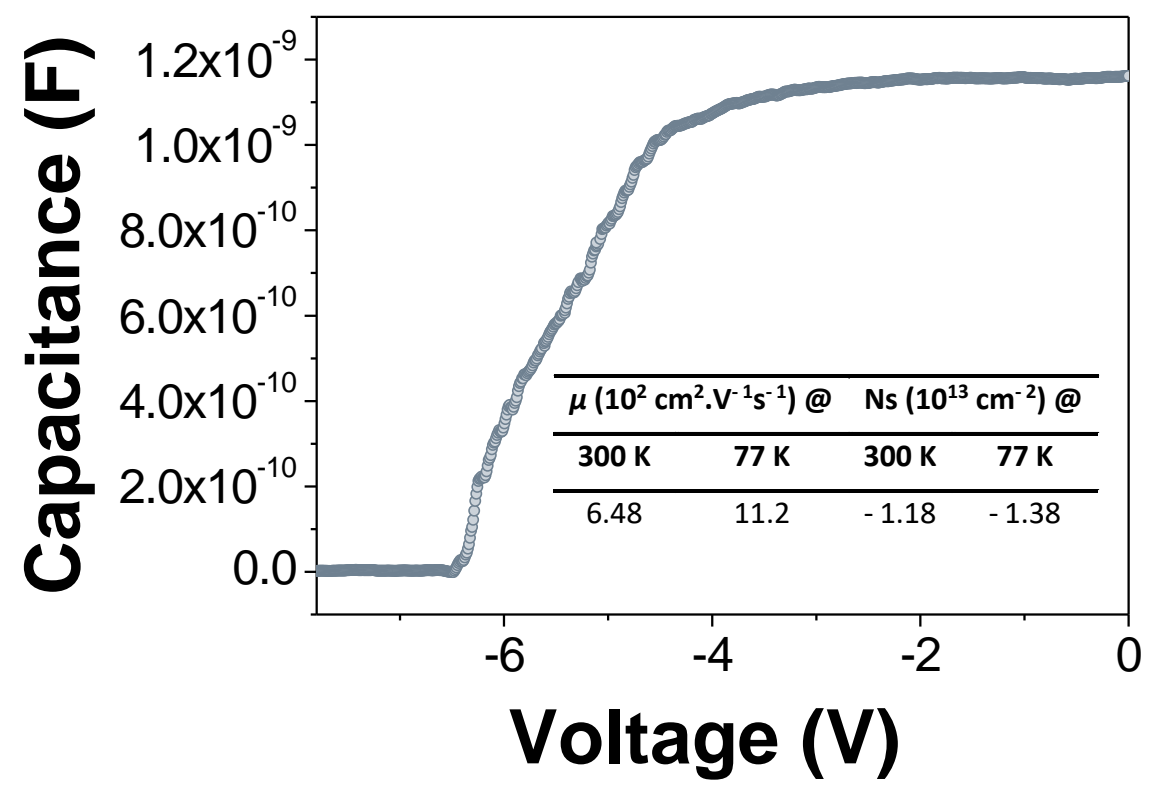

Figure 5 - C-V curve acquired by $\mathrm{Hg}$-probe at a frequency of $20 \mathrm{KHz}$. The inset reports mobility $(\mu)$, and charge density values $(N s)$, as obtained by Hall measurements at 300 $\mathrm{K}$, and $77 \mathrm{~K}$.

\section{Conclusions}

The presented data demonstrated that strained GaN/AIGaN heterostructures can be achieved at low temperature $\left(750^{\circ} \mathrm{C}\right)$, with sharp interfaces and epitaxial relationship among the layers, thus enabling the formation of a $2 D E G$, which can be used for device applications. The quality of gallium nitride layer obtained by RPA-MOCVD, where the remote plasma is involved in all the steps of the growth from the in-situ SiC substrate cleaning and nitridation, to AIN, GaN and AIGAN reduced temperature growth meets the state-ofthe-art obtained with the conventional semiconductor growth technologies (MOCVD and MBE). The plasma configuration chosen is remote with respect to the growth surface, similarly to the MBE growth. Namely the substrate is positioned in the plasma afterglow to avoid ion and electron bombardment of the growing front. 
As a step beyond $\mathrm{MBE}$, in our hybrid approach the in-situ $\mathrm{H}_{2}$ plasma cleaning of the SiC substrate is integrated in the growth process. As a step beyond conventional MOCVD, we avoid the use of high flow of $\mathrm{NH}_{3}$, producing it in-situ during the growth with a $\mathrm{N}_{2} / \mathrm{H}_{2}$ plasma activation. For the more critical AlGaN layer, we observe only limited relaxation and a level of strain suitable for the onset of piezoelectric and spontaneous polarisation induced 2DEG with a large carrier density. Optical measurements suggest the presence of defect states in such a layer, which are probably the cause of the still low carrier mobility. Results demonstrate the possibility to achieve HEMT heterostructures without ammonia source and at low temperature, which can be ascribed to the combination of substrate preparation steps, engineered buffer layer, and growth conditions.

\section{Acknowledgments}

Authors are grateful to the project EleGaNTe - PON ARS01_01007 for funding this research work. We are obliged to Prof VM Kaganer, Paul-Drude-Institut für Festkörperelektronik, Berlin (DE), for his helpful and constructive discussions.

\section{References}

1 J. Edmond, A. Abare, M. Bergman, J. Bharathan, K. Lee Bunker, D. Emerson, K. Haberern, J. Ibbetson, M. Leung, P. Russel and D. Slater, J. Cryst. Growth, 2004, 272, 242-250.

2 C. Jia, T. Yu, X. Feng, K. Wang and G. Zhang, Superlattices Microstruct., 2016, 97, 417-423.

3 T. Kabemura, S. Ueda, Y. Kawada and K. Horio, IEEE Trans. Electron Devices, 2018, 65, 3848-3854.

4 V. Tasco, A. Campa, I. Tarantini, A. Passaseo, F. González-Posada, A. Redondo-Cubero, K. Lorenz, N. Franco and E. Muñoz, J. Appl. Phys., 2009, 105, 063510.

5 H. Pedersen, S. T. Barry and J. Sundqvist, J. Vac. Sci. Technol. A, 2021, 39, 051001.

6 Y. Cordier, B. Damilano, P. Aing, C. Chaix, F. Linez, F. Tuomisto, P. Vennéguès, E. Frayssinet, D. Lefebvre, M. Portail and M. Nemoz, J. Cryst. Growth, 2016, 433, 165-171.

7 H. Rabiee Golgir, D. W. Li, K. Keramatnejad, Q. M. Zou, J. Xiao, F. Wang, L. Jiang, J.-F. Silvain and Y. F. Lu, ACS Appl. Mater. Interfaces, 2017, 9, 21539-21547.

8 Y. Lu, H. Kondo, K. Ishikawa, O. Oda, K. Takeda, M. Sekine, H. Amano and M. Hori, J. Cryst. Growth, 2014, 391, 97-103.

9 W. Wang, W. Yang, Y. Lin, S. Zhou and G. Li, Sci. Rep., 2015, 5, 16453.

10 M. Sato, Appl. Phys. Lett., 1996, 68, 935-937.

11 T. Tokuda, A. Wakahara, S. Noda and A. Sasaki, J. Cryst. Growth, 1998, 183, 62-68.

12 M. Losurdo, G. Bruno, A. Brown and T.-H. Kim, Appl. Phys. Lett., 2004, 84, 4011-4013.

13 S. Sugianto, A. Subagio, E. Erzam, R. A. Sani, M. Budiman, P. Arifin and M. Barmawi, J. Math. Fundam. Sci., 2001, 33, 1-4.

14 M. Losurdo, M. M. Giangregorio, G. Bruno, A. Brown and T.-H. Kim, Appl. Phys. Lett., 2004, 85, 40344036.

15 F. Silie, C. Junfang, G. Peng and W. Chun-ann, Vacuum, 2012, 86, 1517-1521.

16 K. S. A. Butcher, B. W. Kemp, I. B. Hristov, P. Terziyska, P. W. Binsted and D. Alexandrov, Jpn. J. Appl. Phys., 2012, 51, 01AF02.

17 G. Meneghesso, F. Rampazzo, P. Kordos, G. Verzellesi and E. Zanoni, IEEE Trans. Electron Devices, 2006, 53, 2932-2941.

18 V. M. Kaganer, O. Brandt, A. Trampert and K. H. Ploog, Phys. Rev. B, 2005, 72, 045423. 
19 M. A. Moram and M. E. Vickers, Rep. Prog. Phys., 2009, 72, 036502.

20 V. M. Kaganer, B. Jenichen, M. Ramsteiner, U. Jahn, C. Hauswald, F. Grosse, S. Fernández-Garrido and O. Brandt, J. Phys. Appl. Phys., 2015, 48, 385105.

21 P. Motamedi and K. Cadien, RSC Adv., 2015, 5, 57865-57874.

22 H. V. Stanchu, A. V. Kuchuk, Y. I. Mazur, C. Li, P. M. Lytvyn, M. Schmidbauer, Y. Maidaniuk, M. Benamara, M. E. Ware, Z. M. Wang and G. J. Salamo, Cryst. Growth Des., 2019, 19, 200-210.

23 D. J. Wallis, D. Zhu, F. Oehler, S. P. Westwater, A. Pujol and C. J. Humphreys, Semicond. Sci. Technol., 2013, 28, 094006.

24 O. Ambacher, B. Foutz, J. Smart, J. R. Shealy, N. G. Weimann, K. Chu, M. Murphy, A. J. Sierakowski, W. J. Schaff, L. F. Eastman, R. Dimitrov, A. Mitchell and M. Stutzmann, J. Appl. Phys., 2000, 87, 334-344.

25 J.-M. Wagner, Phys. Rev. B, , DOI:10.1103/PhysRevB.66.115202.

26 N. Nepal, J. Li, M. L. Nakarmi, J. Y. Lin and H. X. Jiang, Appl. Phys. Lett., 2005, 87, 242104.

27 J. Li, K. B. Nam, J. Y. Lin and H. X. Jiang, Appl. Phys. Lett., 2001, 79, 3245-3247.

28 J. P. Bergman, T. Lundström, B. Monemar, H. Amano and I. Akasaki, Appl. Phys. Lett., 1996, 69, 34563458.

29 D. Jana and T. K. Sharma, J. Phys. Appl. Phys., 2016, 49, 265107.

30 I. Vurgaftman and J. R. Meyer, J. Appl. Phys., 2003, 94, 3675-3696.

31 I. V. Osinnykh, T. V. Malin and K. S. Zhuravlev, Semicond. Sci. Technol., 2015, 30, 085010.

32 J.-P. Zhang, D.-Z. Sun, X.-L. Wang, M.-Y. Kong, Y.-P. Zeng, J.-M. Li and L.-Y. Lin, Appl. Phys. Lett., 1998, 73, 2471-2472.

33 N. Akopian, A. Vardi, G. Bahir, V. Garber, E. Ehrenfreund, D. Gershoni, C. Poblenz, C. R. Elsass, I. P.

Smorchkova and J. S. Speck, Appl. Phys. Lett., 2009, 94, 223502.

34 D. Zanato, S. Gokden, N. Balkan, B. K. Ridley and W. J. Schaff, Semicond. Sci. Technol., 2004, 19, 427-432. 